\title{
Cáncer del tiroides en niños y adolescentes
}

\author{
Dra. Valeska Philippi R. ${ }^{1}$; Dr. Enuique López C. ${ }^{1}$; Dr. José Torçes $\mathrm{O}^{2}$; Dra. Nelly Abodowsky $\mathbf{G}^{3}$; \\ Dr. Roberto Volpato de V. ${ }^{2}$; Dr. Miguel Domínguez E. ${ }^{1}$; Dra. Ximena Vivanco $W^{3}$
}

\section{Thyroid cancer among children and adolescents}

Clinical and pathological features of 36 patients with thyroid cancer under 19 years of age were studied: at histological examination $55.5 \%$ (20 patients) had papillary carcinoma and $44.5 \%$ (16 patients) the follicular typo of neoplasn. No cases of undifferentiated and medullary cancers were detected. Twenty two ( $61 \%)$ cases had single nodule goiters, while in $14(39 \%)$ patients these were multinodular. Nine cases of early metastases to regional lymphatic tissues and 4 of distant metastases were seen. All patients underwent surgical treatment and were given replacement therapy with thyroid hormones. Additionally nineteen patients were treated postoperatively with $\mathbf{l}^{\mathrm{j} 31}$. There were 4 wases of postoperative lyypoparathyroidism, one of inferior laryngeal nerve lesion and one operating room death. Three regional recurrences of thyroid carcinoma were seen. Mean length of follow up was 15.4 (range $]$ to 40) years and included all cases: there was only one death attributable to thyroid cancer. The prognosis and evolution of thyroid cancer in children and adolescents looks rather benigh even in the presence of regional or long distance metastases.

(Key words: thy roid neoplasms, thyroidectomy, thyroid gland, thyroid hormones).

El cáncer de tiroides (CT) en nifĩos y adolescentes es poco frecuente en Chile. En 1983 representaba 5 to del total de las defunciones por tumores malignos y $0,8 \%$ del total de muertes en el país'. Según algunos autores el interés en CT ha ido en aumento en los últimos años debido probablemente a su relación con la irradiación de cabeza, cuello y mediastino superior por causas diversas, técnica que estuvo en boga hace algunos años ${ }^{2,3}$. Por otra parte, también se ha mencionado una mayor incidencia de CT en regiones de bocio endémico, como también en niños con tiroides ectópicos o aplasia de un lóbulo ${ }^{4}$. Además, se ha descrito la relación del CT con un defecto en la sintesis de $\mathrm{T}_{4}$ e hipotiroidismo inadecuadamente tratados y que, por lo tanto, están sometidos a una intensa estimulación con TSH endógeno ${ }^{4,5}$.

Conscientes de la escasa experiencia en CT en niños $y$ adolescentes, $y$ de los pocos trabajos

1. Servicio de Medicina, Departamento de Endocrinología, Hospital San Juan de Dios, Facultad de Medicjna, División Ciencias Médicas Occidente, Universidad de Chile.

2. Departamento de Cirugía, Facultad de Medicina, División Ciencias Médicas Occidente, Universidad de Chile.

3. Servicio de Pediatría y Cirugía Infantil, Facultad de Medicina, Dłvisión Ciencias Médicas Occidente, Universidad de Chile. publicados sobre el tema en nuestro país, hemos querido presentar nuestra experiencia haciendo un análisis descriptivo de algunas caracteristicas clínico-patológicas, el tratamiento y su evolución posterior.

\section{MATERLAL Y METODO}

Fintre mayo de 1954 y diciembre de 1987 se registraron 450 casos de CT en el Departamento de Endocrinología del Hospital San Juan de Dios, Parte de esta expetiencia ha sido publicada por nosotros 6,7 . Hubo sólo 36 pacientes menores de 19 años (8\%), los que constituyen el material de la presente publicación. La edad fluctuó entre 4 y 19 años con un promedio de 15 años. Veinticinco $(69,4 \%)$ correspondieron al sexo femenino y $11(30,6 \%)$ al sexo masculino.

Los pacientes con bocio nodular fueron estudiados clínicamente y con exámenes de laboratorio: cintigra. fía, tisoidea utilizando preferentemente yodo-131 y, ocasjonalmente, tecnecio (99 mTc), ecografía tiroidea, radiografías de cuello $y$ tórax y determinación hor. monal. Empleamos la clasificáción diseñada por la OMS para separar los distintos tipos histológicos de CT. El cáncer diferenciado (papilar y fohcular) fue subdivídido según criterios estándar ${ }^{\mathrm{g}}$ : la forma papilar (CP) en oculto, cuando el tumor fue menor de $1 \mathrm{~cm}$ de diámetro; intratiroideo, cuando el tumor estuvo confinado a los límites anatómicos de la glándula; extratiroideo, cuando el tumot sobrepasó la cápsula del tiraides invadiendo vía área, digestiva, grandes vasos, nervio recurrente y músculos pretiroideos. E] cáncer folicular (CF) se clasificó como encapsulado, cuando el tumor folicular presentó angioinvasión $y, 0$, capsular asslada; invasor, cuando el tumor sobrepasó la cápsula 
tiroidea invadiendo $\mathrm{cl}$ tejido tiroideo vecino, estructuras del cuello o comprometiendo masivamente los vasos sanguíneos peritumorales.

Todos los enfermos, con excepción de dos, fueron operados por nuestro equipo quirúrgico.

Desde 1961 a la fecha utilizanos como terapia contplementaria yodo-131. Se consideró que el paciente fue tratađo con yodo-131 sólo si recibió el radiofármaco dentro de los 12 primeros meses consecutivos a la intervención inicial. El tratamiento complementario con yodo-131 se hizo en forma ablativa (profiláctica) y terapéutica. La dosis ablativa fhuctuó entre $30-80 \mathrm{mCi}$. La dosis terapéutica se utilizó en casos de enfermedad residual, cuando quedó un remanente tiroideo postablación, en casos de recurrencia y cuando hubo metástasis a distancia.

Todos los enfermos fueron mantenidos con dosis supresivas de hormona tiroidea.

Se definió como metástasis regionales la presencia de linfonodos con tumor en la región cervical, comprobados por biopsia previa o durante el acto operatorio. Si su presencia fue detectada antes de 2 meses de la intervención quirúrgica, se definió también como metástasis inicial. La presencia de metástasis a distancia se interpretó como la existencia de un tumor fuera de la zona tiroidea, del cuello o del mediastino superior.

Las recurrencias fueron calificadas corno: local, en la región tireidea; regional, en los tinfonodos cervicales y a distancia, si la enfermedad reapareció en pulmones. hueso o cerebro.

Consideramos mortalidad operatoria cuando el paciente falleció antes de 30 dias de la operación. Consideramos a los pacientes muertos por CT cuando hubo signos de enfermedad activa en su último control antes de fallecer. El resto de los pacientes fue catalogado como vivo sano, vivo con enfermedad local o a distancia y muet to por otra causa,

\section{RESULTADOS}

Todos los casos analizados fueron carcinomas diferenciados, no hubo casos de cáncer medular ni anaplásico. Veinticinco pacientes $(69 \%)$ correspondieron al sexo femenino (relación $F: M=2,3: 1$ ).

El tipo histológico correspondió en 20 casos a CP $(55,5 \%)$ y en 16 a CF $(44,5 \%)$. Los casos de CP se distribuyeron en ocultos $(n=2)$, intratiroideos $(n=13)$, incluye un quiste tirogloso, $y$ extratiroideos $(n=5)$. Los $C F$ se distributyeron en: encapsulados $(n=10)$ e invasores $(n=6)$.

En la tabla 1 se aprecia la distribución de CT por gupos etarios y tipo histológico. Hubo solamente 5 casos menores de 10 arfos. La edac a la primera consulta osciló entre los 4 y 19 años promedio 15 años.

El motivo de consulta fue bocio nodular er: 28 casos; la aparición de adenopatjas cervicales en 7 casos y metástasis pulmonares en un caso. El tiempo transcurrido entre la aparición de sín-
Tabla 1

Distribución por grupo de edad y tipo histológico en 36 casos de cáncer del tíroides. Hospital San Juan de Dios, 1954-1987

\begin{tabular}{lccc}
\hline Edad & Papilat & Folicular & Total \\
\hline $0-5$ & - & 1 & 1 \\
$6-10$ & 3 & 1 & 4 \\
$11-15$ & 2 & 7 & 9 \\
$16-20$ & 15 & 7 & 22 \\
TOTAL & 20 & 16 & 36 \\
\hline
\end{tabular}

tomas y la primera consulta fue, en promedio, de 18 meses. Un solo paciente con CF dio el antecedente de irradiación de la cara por hemangioma del labio. Respecto al tipo de bocio, éste fue uninodular en 22 casos (63\%) y multinodular en 14 casos $(37 \%)$. El estudio cintigráfico mostró 22 nódulos fríos, 2 funcionantes y un bocio multinodular presentó nódulos funcionantes e hipofuncionantes. En 11 pacientes no hizo cintigrama porque no contábamos aún con yodo-131.

Metástasis regionales iniciales hubo en 9 casos (25\%), 4 en CP y 5 en CF. Se detectaron metástasis pulmonares iniciales, uno de los cuales presentó, además, metástasis óseas ( 3 en CP y 1 en CF), en 4 casos.

El tipo de intervención quirúrgica practicada fue: en un caso resección de quiste tirogloso, tiroidectomia incluyendo el istmo en 11, tiroidectomía subtotal bilateral en 13, tiroidectomía total o casi total en 6 pacientes.

La disección cervical se practicó en 11 casos y fue de 2 tipos: radical, que incluyó la extirpación del músculo esternocleidomastoideo, vena yugular y nervio espinal en 3 casos y modificada, cuando se preservaron dichas estructuras, en 8. En 3 pacientes fue preciso hacer una disección cervical bilateral que se practicó en un segundo tiempo, debido al compromiso de linfáticos regionales. Un paciente sufrió lesión operatoria del nervio recurrente por estar comprometido por el tumor y 4 casos tuvieron hipoparatidoidismo, 3 de ellos secundario a tiroidectomía total y 1 a tiroidectomía subtotal. Todos habian sido sometidos a vaciamiento radical.

Hubo un solo caso de mortalidad operatoria, se trataba de un niño de 4 años de edad con CF invasor que comprometía severamente la vía respiratoria y tenía metástasis óseas y pulmona- 
res. El tumor fue sólo parcialmente resecable y el paciente falleció 15 dias después de la operación de bronconeumonía.

Se dio tratamiento complementario con yodo-131 en 19 casos, con dosis que fluctuaron entre 50 y $330 \mathrm{mCj}$ con un promedio de $110 \mathrm{mCi}$. En 7 pacientes se dio yodo. 131 varios años después de la intervención.

Hubo 3 pacientes con recurrencia local (3 CP intratiroideos) a $\operatorname{los} 2 ; 4$ y 20 años, respectivamente. Todos fueron reoperados practicándose tiroidectomía total y dando luego yodo-131. Actualmente se encuentran todos vivos, sanos y libres de enfermedad. En cuatro casos se detectaron metástasis pulmonares iniciales ( 3 con $\mathrm{CP}$ y 1 con CF). Un niño de sexo masculino de 10 antos con un CP intratiroideo fue sometido a tiroidectomía total y se le suministró una dosis ablativa de yodo-131; el paciente desapareció de control por 18 años, reapareciendo con mixedema, acentuado retraso del desarrollo físico y extensas metástasis pulmonares y óseas que no respondieron al tratamiento con más de $300 \mathrm{mCi}$ de yodo-131 en 2 dosis, falleciendo al poco tiempo. De los otros dos pacientes con CP y metástasis pulmonares iniciales, uno está sano, sin eviäencias de enfermedad 20 años después del tratamiento, y otro tiene signos de inactividad de sus lesiones pulmonares que parecen de tipo fibroso y no captan radioyodo 30 años después del tratamiento. Cabe mencionar que estos 2 pacientes habian sido diagnosticados erróneamente como tuberculosis pulmonares, recibiendo durante varios meses la terapia correspondiente.

Hubo sólo 4 fallecimientos en nuestra casuística, uno por muerte operatoria, 2 por causas ajenas a la enfermedad y sólo uno debido al CT (CT intratiroideo).

El tiempo promedio de control de nuestros pacientes ha sido de 15,4 años, rango de 1 a 40 años. No hay ningún paciente perdido definitivamente de control, lo cual da un seguimiento de $100 \%$.

\section{DISCUSION}

A pesar que el CT es la neoplasia endocrina más frecuente, constituye menos de $1 \%$ de todas las neoplasias ${ }^{5,9}$. No hay publicaciones chilenas sobre CT en niños y adolescentes. Eggers ${ }^{4}$ encontró sólo $4 \mathrm{CT}$ en 11 casos de bocios operados entre 3 y 13 años de edad. En una publicación de 224 bocios nodulares operados en el Hospital
San Juan de Dios entre 1979 y 1981 se encontraron 16 casos $(7,1 \%)$ en menores de 19 años, 6 de éstos eran $\mathrm{CT}^{10}$.

Se ha descrito mayor frecuencia de CT en mujeres que en hombres $2,5,11$, con relaciones de 2:1. En nuestra serie $e^{6,12,13}$ la proporción fue de 2,3:1. También en nuestros casos encontramos mayor incidencia de CT en la segunda década. La razón reside fundamentalmente en la larga historia natural de la enfermedad ${ }^{3,8,14}$.

En contraste con Winship ${ }^{2}$, que menciona el antecedente de irradiación en $80 \%$ de los casos, nosotros lo encontramos sólo en uno, lo que se debe posiblemente a la difícil obtención de este dato en nuestro medio $y$ a la edad de la muestra pues dicha técnica se usó especialmente hasta hace 2 décadas entre 1940 y 1955.

La incidencia de CT es alta en pacientes menores de 20 años con bocio nodular, siendo de 40 a $70 \%$ en núfos con nódulo solitario ${ }^{2,15,16}$. Por lo tanto, en los niños con nódulos tiroideos la conducta terapéutica đebe ser más agresiva que en adultos jóvenes.

Los pacientes descritos representan $8 \%$ del total de carcinomas de la glándula tiroides atendidos en nuestro hospital, cifra inferior a la de $15 \%$ citada por algunos autores, excluidos los nif̉os expuestos a irradiación ${ }^{16,17}$, y similar a otras descripciones ${ }^{5,9}$. Por otra parte, la elevada prevalencia del CF $(44,5 \%)$ en niffos es claramente superior a la que encontramos para el conjunto de todas las edades ${ }^{\circ}(32,6 \%)$, lo que también ha sido mencionado por otros, $s^{5}$. Si bien nosotros hemos visto una clara disminución del CF en los últimos años $s^{6,7}$ la prevalencia en el nifio no ha varado $^{16}$. La posibilidad de sobrediagnosticar CF encapsulado en pacientes provenientes de zonas de endemia de bocio como lo sugiere Fransilla ${ }^{18}$ ha sido analizada por nosotros y creemos haber clarificado este hecho en nuestro material histológico, dado que fue revisado en dos oportunidades por 2 grupos distintos de nuestros patólo$\operatorname{gos}^{6,7}$.

El CT en niffos se caracteriza por dar precozmente metástasis regionales y a distancia ${ }^{2,3,9,19}$; sin embargo, el compromiso ganglionar no se re. laciona con el tamaf́o del tumor primitivo y el pronóstico" e incluso algunos autores han estimado que su presencia sería índice de mejor pronóstico ${ }^{8,20,21}$, el que, sin embargo, estaría más bien relacionado con la edad de los pacientes $^{22}$. Todos ruestros enfermos que tenian linfonodos positivos como única manifesta- 
ción de diseminación tumoral están actualmente vivos y sanos confirmando esta aseveración. A pesar de que el CT predomina en el sexo femenino, los de sexo masculino presentan formas más agresivas, lo que fue corroborado por nosotros por el hallazgo de metástasis pulmonares exchusivamente en nifíos de sexo masculino. Hasta $20 \%$ de los casos pueden tener metástasis pulmonares iniciales que frecuentemente se confunden con procesos infecciosos del pulmón ${ }^{23}$, como sucedió en 2 de nuestros pacientes.

El comportamiento relativamente benigno del CT en niños no debe inducir a terapéuticas limitadas, sino a conductas similares a las empleadas en adultos ${ }^{24}$. Con respecto al tratamiento quirúrgico, algunos autores han preconizado la tiroidectomia total ${ }^{23,25,26}$. Este criterio no es compartido por nosotros ni por grandes series extranjeras $^{8,18,27,30}$. Los autores que proponen la tiroidectomía total se apoyan en la multifocalidad del $\mathrm{CP}$. El compromiso contralateral parece ocurrir en $30 \%$ de los casos ${ }^{31}$.

La cirugía agresiva radical va unida a mayor incidencia de hipoparatiroidismo, especialmente cuando se hace vaciamiento cervical bilateral, que en algunas series llega a 20 y $27 \% 5,12,14,32$. Nosotros tuvimos sólo cuatro casos, tres habian sido sometidos a tiroidectomía total y todos a vaciamiento cervical bilateral.

Nuestra actitud quirúrgica depende del grado de extensión anatómica del tumor. Reservamos la tiroidectomía total para los CP extratiroideos, los CF invasores y para cualquier variedad de Ca diferenciado que tenga metástasis a distancia en el momento del tratamiento quirúrgico inicial. La tiroidectomía subtotal (casi total) se indica en los CP intratiroideos. La lobectomía con istmectomía se limita a los CF encapsulados y CP ocultos. La disección cervical radical ha sido sustituida por la disección modificada que no tiene las desventajas cosméticas, funcionales $y$ psicológicas de la primera, cumpliendo con los mismos propósitos ${ }^{1,6,33}$.

También existe controversia sobre la conve. niencia de usar yodo-131 en forma sistemática. En general, quienes apoyan la tiroidectomía total o casi total son también partidarios del uso de yodo-131 2, 23,25. Por el contrario, los que preconizan cirugía más conservadora emplean radioyodo en forma más selectiva ${ }^{8,20,32,27,30}$, criterio que compartimos, pues no usamos yodo-131 en el CP oculto ni en el CF encapsilado o minimamente invasor. Las metástasis re- gionales no constituyen indicación de yodo-131 para nosotros.

El CT en niños suele presentar precozmente metástasis regionales que pueden estar disem:nadas a distancia en el momento de consultar. La presencia de linfonodos, sin embargo, no tiene repercusión pronóstica y las metástasis pulmonares pueden eventualmente desaparecer con yodo-131 y homnonas tiroideas.

El CT en nitos y adolescentes se caracteriza por su excelente pronóstico ${ }^{20,34}$, con una esperanza de sobrevida similar a la población nor$\mathrm{mal}^{5,9,12,24,34}$. Nosotros tuvimos sólo un caso fallecido por CT, lo que corrobora esta afirmación.

El CF en niños tiene mejor pronóstico que en adultos porque en éstos hay progresión a las formas indiferenciadas, lo que no sucede en los primeros. Por otra parte, se ha comprobado que CT en nifín, especialmente el CP, es más sensible al yodo- $131^{35}$, como también el tiroides infantil es más sensible al estimulo carcinógeno determinado por el aumento de TSH endógeno ${ }^{9,19}$, por to que es fundamental el tratamiento con homnonas tiroideas.

\section{RESUMEN}

Se estudian las características clínjco-patológicas de 36 pacientes menores de 20 anos portadores de cáncer del tiroides (CT).

Encontramos $20(55,5 \%)$ cáncer papilar y 16 $(44,5 \%)$ cáncer foliculat. No hubo cáncer medular $\mathrm{ni}$ anaplástico. Veintidós $(61 \%)$ casos presentaron bocio uninodular y 14 (39\%) multinodular. Hubo 9 pacientes con metástasis linfáticas injciales y 4 a distancia. Todos fueron operados y tratados con hormonas tiroideas. Diecinueve recibieron tratamiento complementario con yodo-131.

Morbilidad quirúrgica: 4 hipoparatiroidismo y una lesión del nervio recurrente. Muerte operatoria: 1. Recurrencias de CT: 3 regionales.

El promedio de años de control fue de 15,4 años (rango 1 a 40) con un seguimiento de 100\%. Hubo una recumencia por CT.

Se concluye en la benignidad evohtiva del CT en niños y adolescentes aun cuando haya metástasis regionales y a distancia.

\section{REFERENCIAS}

1. Ministerio de Salud de Chlle: Anuario de Defunciones y Causas de Muerte, 1983; pp. 57. 
2. Winship $T$., Rosvol R.: Child hood thyroid carcinoma, Cancer 1961; $14: 434-442$.

3. Haylis A., Kennedy R., Beahrs $O$.: Management of the child with thyroid carcinoma. JAMA 1960; 173: 21-28.

4. Eggers $M$., Beas $F_{\text {.: }}$ Nódulo tiroideo en el nin̄o. Rev Chil Pediatr 1982; 53: 456-459.

5. Buckwalter $J$., Thomas $C$., Freeman $f$ : Is childhood thyroid cancer a lethal disease. Ann Surg $1975 ; 181: 632-639$.

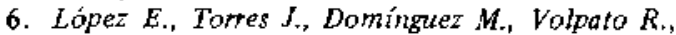
Martinez V., Maina J., Rolondo M., Lobo G., Galarza S., Pêrez G, y* Philippi V.: Cáncer del tiroides: Aspectos clínicos de 320 casos recolectados en 27 años. Rev Med Chil 1982; 110: 123-132.

7. Torres J., Volpato R., Power E, Lobez E, Dominguez M., Maira J., Ugarte J.M. and Martinez V.: Thyroid cancer: Survival in 148 cases followed for 10 years or more, Cancer $1985 ; 56 ; 2298-2304$.

8. Woolner L., Beahrs O., Black M., Mc Conckey W. and Keoting F. Jr.: Classification and prognosis of thyroid carcinoma. A study of 885 cases observed in a thirty year period. Am J Surg 1961; 102: 354.387 .

9. Buckwalter J. Guill $N$., Thomas $C$.: Cancer of the thyroid in youth. World J Surg 1981; 5: 15-25.

10. Venegas C., López E. y Cerpa F.: Patología tírojdea médico-quirúrgica: análișis de la experiencia de 3 años (1979-1981). Bol Hosp SJ de Dios 1983; 30: $303-307$.

11. Herzog B.: Thyroid gland diseases and tumours surgical aspects. Prog Pediatr Surg 1983; 16: 15. 22.

12. Geopfert H., Dichtel W., Somoon N.: Thyroid cancer in children and teenagers. Arch Otolaryngol Head Neck Surg 1984; 110-72-75.

13. Shields W., Alvizoturi M. Colcock B.: Carcinoma of the thyroid in childhood and adolescence. Can. cer 1953;6: $1139-1146$.

14. Leeper $R_{1}$ : Controversies in the treatment of thyroid cancer. The New York Memorial Hospital Approach. Thy roid Today $1982 ; 4 ; 1.4$.

15. Scott $M$., Crawford $J$.: Solitary nodules in childhood. Is the incidence of thyroid carcinoma declining. Pediatrics 1976;58: 521-525.

16. Ralison M., Dobyns B., Keating F. et al.: Thyroid nodularity in children. JAMA 1975; 233: 1069 . 1072.

17. Silvermon $S$., Nussboum M., Rousen A.: Thyroid nodules in children: a ten year experience at one institution. Mt Sinai J Med (NY) 1979; 46;460463.

18. Fransitla $K$ : Value of histological classification of thyroid cancer. Acta Pathol Microbiol Immunol Scand (A) 1971; Suppl. 225: 5-76.

19. Cloyton G., Kirkland R.: Cances of the thyroid in children in Greenfield L.D. (editor). Thyroid Cancer, Boca Raton, Fla. C.R.S. Press Inc. 1978.
20. Cady B., Sedgwick C., Meissner W., Wool M., Salzman $F$, and Werber $J_{\text {.: }}$ Risk factor analysis in differentiated thyroid cancer. Cancer 1979:43: $810-820$.

21. Cady B., Rossi R., Silverman M., Wove M.: Further cvidence of the viability of risk group defirition in differentiated thyroid carcinoma. Surgery 1985; 98: $1171-1178$.

22. Rossi R., Nieroda C., Cady B., Wove M.: Malignancies of the thyroid gland. The Lahey Clinic experience. Surg Clin N A $1985 ; 65 ; 211-230$.

23. Harness J., Thompson N., Mc Leod M. Eckhauser $F$. and Lloyd R.: Follicular carcinoma of the thyroid gland: Trends and treatment. Surgery 1984; 96: 972-980

24. Joppjch J., Rohrer H., Hecker W., Knorr D., Doum $R$ : 'Thyroid carcinoma in childhood. Prog Pediatr Surg 1983; 16: 23-28.

25. Samoon $N$, Maheshwari $Y$., Hill $C$. Jr. Schultz $P$. Hoynic T., Hickey R., Clark R., Goepfert H., Jbónez $M$. and Liton $C$.: Impact of therapy for differentiated carcinoma of the thyroid. An analysis of 706 cases. J Ctin Endocrinol Metab 1983; 56: 1131-1138.

26. Young R., Mazzaferri E., Rahe A. and Dorfman $S$.: Purc follicular thyroid carcinoma impact of therapy in 214 patients. J Nucl Med 1980:21: 733-737.

27. Crile G. Jr.: Changing and tesults in patients with papillary carcinoma of the thyroid. Surg Gynecol Obstet 1971 ; 132: 460-468.

28. Betrgie J., Brown C., Donach J. and Richardson J.: Primary malignant tumours of the thyroid. The relationship between histological classification and clinical bchaviour. Br J Surg 1976; 63: 173-181.

29. Thompson $N$. and Horness $J$.: Complication of total thyroidectorny for carcinoma. Surg Gynecol Obstet 1970;131: 861-868.

30. Mc Conahey W'., Hay J., Woolner L., Van Heerden J. and Taylor $\boldsymbol{W}$.: Papillary thyroid cancer treated at the Mayo Clinjc, 1946 through 1970. Initial manifestations. Pathological findings. Therapy and outcome. Mayo Clin Proc 1986; 61: 978-996.

31. Tollefsen R., Schah Y., Huros A.: Papillary carcinoma of the thyroid gland after initial surgical treatment. Am J Surg 1972; 124:468.472.

32. Anderson A., Bergdoht l.., Boquist L.: Thyroid carcinoma in childsen. Am J Surg 1977; 43: 159 . 163.

33. Richardson J., Beaugié J., Brown C., Domach J.: Thyroid cancer in young patients in Great Britain. Brit J Surg 1974;61: 85-89.

34. Maheshwart Y., Hill $C$., Haynic et al.: $1^{131}$ therapy in differentiated thyroid carcinoma. Cancer 1981; 47: 664-671.

35. Mazaferri E. and Young R.: Papillary thytoid carcinoma: a ten year follow-up report of the impact of therapy in 576 patients. Am J Med 1981;70: $511-518$. 